\title{
Erratum to: Meta-analytic support vector machine for integrating multiple omics data
}

SungHwan Kim ${ }^{1,2}$, Jae-Hwan Jhong ${ }^{1}$, JungJun Lee ${ }^{1}$ and Ja-Yong Koo ${ }^{{ }^{*}}$

${ }^{*}$ Correspondence: jykoo@korea.ac.kr

'Department of Statistics, Korea University, Anam-dong, 136-701 Seoul, South Korea

\section{Erratum}

After publication of the original article [1], the author noticed errors to Table 1. The errors occurred in the fourth and seventh columns labelled 'Youden'. The correct version of Table 1 is included in this erratum.

\section{Author details \\ 'Department of Statistics, Korea University, Anam-dong, 136-701 Seoul, South Korea. ${ }^{2}$ Department of Statistics, Keimyung University, Dalseoku, 42601 Daegu, South Korea.}

Received: 5 February 2017 Accepted: 5 February 2017

Published online: 14 February 2017

\section{References}

1. Kim SH, Jhong JH, Lee JJ, Koo JY. Meta-analytic support vector machine for integrating multiple omics data. BioData Mining. 2017;10:2. doi:10.1186/s13040-017-0126-8. 
Table 1 Shown are the results of experimental studies to compare the meta-logistic model with the meta-analytic SVM

\begin{tabular}{|c|c|c|c|c|c|c|}
\hline \multirow[b]{2}{*}{ Variance (R) } & \multicolumn{3}{|l|}{ Meta-SVM } & \multicolumn{3}{|c|}{ Meta-logistic regression } \\
\hline & Sensitivity (SE) & Specificity (SE) & Youden & Sensitivity (SE) & Specificity (SE) & Youden \\
\hline & \multicolumn{6}{|c|}{ No inclusion of random study } \\
\hline 0.1 & $0.828(0.001)$ & $0.9843(0)$ & 0.812 & $0.1073(0)$ & $1(0)$ & 0.107 \\
\hline 0.3 & $0.8127(0.002)$ & $0.8707(0.001)$ & 0.683 & $0.2087(0.001)$ & $0.996(0)$ & 0.205 \\
\hline \multirow[t]{2}{*}{0.5} & $0.76(0.002)$ & $0.867(0.001)$ & 0.627 & $0.2633(0.001)$ & $0.9123(0.001)$ & 0.176 \\
\hline & \multicolumn{6}{|c|}{ Inclusion of one random study } \\
\hline 0.1 & $0.8007(0.011)$ & $0.9837(0.002)$ & 0.784 & $0.102(0.004)$ & $0.997(0.001)$ & 0.099 \\
\hline 0.3 & $0.6673(0.013)$ & $0.8497(0.009)$ & 0.517 & $0.2113(0.009)$ & $0.966(0.005)$ & 0.177 \\
\hline \multirow[t]{2}{*}{0.5} & $0.6013(0.017)$ & $0.852(0.009)$ & 0.453 & $0.2527(0.01)$ & $0.8667(0.008)$ & 0.119 \\
\hline & \multicolumn{6}{|c|}{ Inclusion of two random studies } \\
\hline 0.1 & $0.624(0.016)$ & $0.9737(0.009)$ & 0.598 & $0.0847(0.005)$ & $0.994(0.001)$ & 0.079 \\
\hline 0.3 & $0.51(0.016)$ & $0.8433(0.006)$ & 0.353 & $0.1727(0.011)$ & $0.9317(0.005)$ & 0.104 \\
\hline 0.5 & 0.4167 (0.012) & 0.85 (0.009) & 0.267 & $0.256(0.012)$ & $0.8193(0.006)$ & 0.075 \\
\hline
\end{tabular}

